\title{
Integration of an automated active control system in building glazed facades for improving sound transmission loss
}

\author{
B. Naticchia, A. Carbonari
}

\begin{abstract}
In buildings, windows and glazed facades are often the weak link in protecting the interior from exterior noise. Since passive means for improving the Sound Transmission Loss (STL) of glazed facades are very expensive and are effective only at high frequencies, an active controller that increases the STL in the low frequency range is an attractive approach to reducing the noise level in buildings with glazed facades, guaranteeing the performance required by the 89/106/CEE European Directive, which made protection against noise a compulsory requirement for buildings. Several kinds of active control system of Sound Transmission were recently studied, whose two main applications are known as Active Noise Control (ANC) and Active Structural Acoustic Control (ASAC) systems. This paper concerns the second approach: it is based on the reduction of structural vibration, through the use of actuators bonded on the vibrating surfaces, driven by an automated control system, whose task is minimizing those vibrations, and consequently, radiated sound. A technological study aimed at choosing a suitable solution for the integration of such automated control systems in buildings with glazed facades is studied, and future research developments are planned.
\end{abstract}

Index Terms - ASAC system, Sound Transmission Loss, building glazed facades, piezoceramic actuators, stack actuators, feedback control.

\section{INTRODUCTION}

$\mathrm{T}_{3}$ HIS paper concerns the preliminary development of an automated control system, aimed at improving the Sound Transmission Loss (STL) of glazed building facades. The 89/106/CEE European Directive, has made protection against noise a compulsory requirement for buildings. In fact, glazed facades often represent the main problem, as they can respect the strict limits imposed by national regulations only using expensive solutions. The two main types of passive means for

B. Naticchia is Full Professor of "Building Construction Management" and "Detail Design of Building" at the Polytechnic University of Marche, in the Faculty of Engineering, Department of Architecture, Construction and Structures, via delle Brecce Bianche, 60131, Ancona, ITALY (e-mail: b.naticchia@univpm.it ).

A. Carbonari, is PhD student at the Polytechnic University of Marche, in the Faculty of Engineering, Department of Architecture, Construction and Structures, via delle Brecce Bianche, 60131, Ancona, ITALY (Corresponding author; phone: +39 0712204397; fax: +39 0712204582; e-mail: alessandro.carbonari@univpm.it ). improving STL presently known are:

- laminated glass technology;

- double glazing.

Both of them can be useful for reducing noise transmission at high frequencies: in particular the laminated solution is used to shift the coincidence effect at very high frequencies improving the STL in a range included between 1500 and $6000 \mathrm{~Hz}$, but this method does not function in the range of low frequencies. On the other hand, in double glazing the coupling between the glass panels and the air layer increases the resonance effect at frequencies lower than $500 \mathrm{~Hz}$. In any case, the low frequency STL can be improved through the adoption of an active control system, capable of controlling the vibrations of glazed panels, while avoiding the use of very expensive glazed facades equipped with massive passive means. In [1] several approaches for active control of double panels are described: in some cases they are based on the production of a canceling wave that interferes destructively with the disturbing one; in other cases control is performed acting directly on the vibration field of structures radiating noise (ASAC). Other researchers [2] apply actuators on a double glazed window in order to control its vibrations: they suggest the use of a feedback controller, which only uses sensors and actuators in the cavity of the double glazed window, thus overcoming the problems tied to the placement of noise sensors outside the windows, suggested by [3] and [4], which is practically infeasible. This solution also requires the use of glazed facades having thick cavities which allow the insertion of loudspeakers within the same. For this reason, when the cavities are narrow, it is convenient to apply both reference sensors and actuators on the same glazed panel, as required for the implementation of a feedback controller: automotive companies as well as plane manufacturers have paid great attention to this approach. In [5] control experiments are performed in a test section comprising the aft portion of a furnished DC-9 aircraft, demonstrating the potential of ASAC in reducing cabin noise in realistic aircraft structures. Using a typical feed-forward controller arrangement driving two shakers as control forces, the researchers achieved global sound level reductions of up to 9 dB. In [6] an aluminum cylindrical test section with a removable floor structure was used to simulate an aircraft fuselage environment. Piezoelectric (PZT) patches were 
bonded directly to the cylinder surface and the system was excited acoustically with an exterior loudspeaker noise source. Using two microphones as error sensors, the ASAC system provided global attenuations in the order of $10 \mathrm{~dB}$ in the cylinder interior. Active control tests were performed by [7] in the cabin of a Cessna fuselage, a typical mid-sized business jet. PZT actuators bonded to the fuselage skin were used as control actuators, to reduce interior noise due to harmonic structural disturbance applied at the engine mount. Using four actuator arrays, each consisting of four patches wired in series, and four error microphones, control was applied to acoustic resonance and off-resonance cases. In the acoustic resonance case, noise reductions of up to $20 \mathrm{~dB}$ were reached.

As discussed further in this paper, the application of secondary sources as actuators to control glazed facade vibrations is more difficult than on metal sheet panels, due to the low degree of controllability that standard actuators (like lead zirconium titanate or PZT) have on glass panels, and to the need to not interfere with their primary function, namely to gaze through them. For this reason, an accurate analysis of several types of actuators is carried out in this paper, as it is the introductory step before the implementation of an automated control algorithm.

This paper is organized as follows: chapter 2 deals with the analysis of the state of art relative to standard kinds of curtain walls. It aims at elaborating an opportune numerical model for the simulation of vibrations induced by plane waves on such glazed facades; chapter 3 deals with the description of an automated ASAC system which can be integrated in buildings; chapter 4 is dedicated to the problem of choosing the right kind of actuators and their proper positioning; capable of guaranteeing high levels of controllability on glass panels, as a consequence of the technological solutions adopted for their application on glazed facades. Concluding remarks and suggestions for future research as the final part of this paper.

\section{TECHNOLOGICAL TASKS FOR THE CONTROL SYSTEM DESIGN}

\section{A. Glazed enclosures to be actively controlled}

A preliminary analysis of the most common glazed curtain walls available for buildings will help to address the study developed in this paper in order to list the requirements and the constraints imposed on the control system by the technological requirements of such building elements. In this paper, glazed facades with punctual anchoring will not be considered, while the analysis will be carried out on ones fixed using linear constraints. This second category can be divided into two main kinds of facades:

- the structural glazing curtain wall;

- the standard glazed facade.

In the first case, glass panels have a structural task, not present in the second case, which will be considered in this contribution. Glass panels can be fixed to a bearing metal frame using one of the following two basic technologies (Fig. 1):

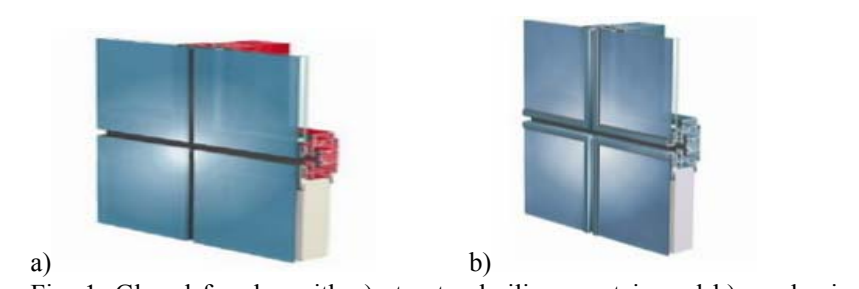

Fig. 1. Glazed facades with a) structural silicone retain and b) mechanical retain.

\section{- structural silicone;}

- mechanical support.

The choice between these two possibilities depends on the image that the designer wants to give to buildings, as in the first case there is the presence of the structural frame on the outside. The study proposed in this paper is relative to simply supported glass panels along their edges, that can represent a good approximation of glazed panels with mechanical retains, that are the most commonly used in the field of construction.

\section{B. Effect of the disturbance on glazed panels}

Considering that the main types of disturbing noise for buildings is produced by road and railway traffic, panels excited by plane waves will be studied in this paper. Analytical solutions for forecasting the behavior of unbounded non flexible panels are known [8], and they can be easily extended to partitions between two rooms or to building envelopes. The STL of glass alone at low frequencies is determined by the panel's static stiffness. Since glass reacts best to excitation frequencies that match its natural frequencies, the low internal damping of tempered glass produces resonances that dramatically decrease STL. Above the resonant frequencies, sound transmission follows the mass law of acoustics and is dictated by the mass or surface density of glass. However, glass has a specific coincidence frequency at which the speed of incident acoustical waves matches that of the glass bending wave. At the coincidence frequency, the acoustic wave is especially effective at causing glass to vibrate, which makes the vibrating glass an effective sound radiator at that specific frequency and above. As a result, glass becomes less of a barrier to sound and exhibits a transmission loss dip, or so-called coincidence dip (in addition to resonance dip at lower frequencies). As stated in chapter I, passive means for improving the transmission loss of glazing systems are effective only at high frequencies shifting the coincidence effect at higher and less effective frequencies, while in the range contained between 60 and $500 \mathrm{~Hz}$ the Sound Transmission Loss remains significantly low.

Analyses concerning the sound power level of road traffic noise were carried out [9], as function of frequency and speed, the results are illustrated in Fig. 2. The data takes into account the contributions of passenger cars, dual axial vehicles and multi-axial vehicles to the summation of the total traffic noise. It can be noticed that there is a high contribution of sound power belonging to the low frequency range, where glazed facades have low STL, even if equipped with special passive means. For this reason, if we want to avoid adopting very thick glass panels, the adoption of an active control system is necessary. 


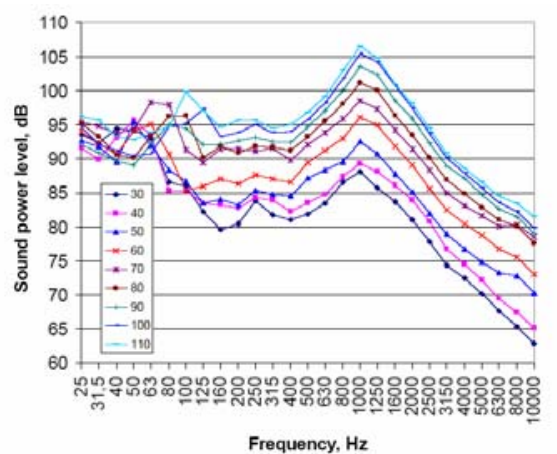

Fig. 2. Equivalent omni-directional sound power level of road traffic noise as function of speed.

\section{Numerical model for the simulation of vibrations induced by plane waves}

The numerical model described in this subsection will be used in chapter IV to analyze the vibrations of glass plates subject to a low frequency disturbance.

As flexural waves in structures are the main responsible for sound emission [10] and they are induced in plates by external disturbances, it is opportune to analytically describe the flexural response of a simply supported plate to a harmonic excitation of arbitrary distribution like $f(x, y, t)=F(x, y) \cdot e^{i \omega t}$ (where $\omega=2 \pi v, v$ is the excitation frequency, $F(x, y)$ is the excitation amplitude):

$E I\left(\frac{\partial^{4} w}{\partial x^{4}}+2 \frac{\partial^{4} w}{\partial x^{2} \partial y^{2}}+\frac{\partial^{4} w}{\partial y^{4}}\right)+\rho h \frac{\partial^{2} w}{\partial t^{2}}=-F(x, y) e^{i \omega t}$

being $i$ the complex unit, $w$ the transversal displacement, $E, I$, $\rho$ and $h$ the elasticity modulus, moment of inertia, density and thickness, respectively, of the plate. One of the possible solutions of (1) for the formalization of the transverse displacement field is obtained describing it as the superposition of infinite modes of the free response of the plate, which is vibrating at the forcing frequency. If we choose a two dimensional coordinate reference system, and $w$ indicates the transversal displacement at the generic point of the glass panel, the solution is given by:

$$
w(x, y)=\sum_{m=0}^{\infty} \sum_{n=0}^{\infty} W_{m, n} \Psi_{m, n}(x, y) e^{i \omega t}
$$

where $W_{m, n}$ are the modal amplitudes that is the maximum displacement at the free natural mode $(m, n) . \Psi_{m, n}$ are the modal shapes, which take into account the shape of the mode $(m, n)$ on the plate, while the exponential term is aimed at describing the dependence with the time and the influence of disturbing frequency. In order to study the transversal displacement motion of a plate whose side lengths are $a$ and $b$, it is necessary to compute the displacement distribution field given by an incident plane wave. Equation (2) is generally approximated in another form, which considers only the superposition of a finite number of vibrating modes, given by the combination of two finite numbers $M$ and $N$ (the validity of the approximation is dependent on the choice of these two values). Thus the displacement distribution is given by [11]:

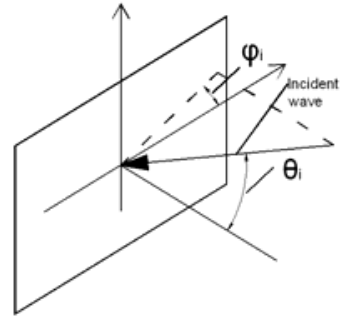

Fig. 3. Angles individuating the direction of incidence of an acoustic plane wave into a glazed plate.

$$
w(x, y, t)=\sum_{m=1}^{M} \sum_{n=1}^{N} W_{m, n} \operatorname{sen}\left(k_{m} x\right) \cdot \operatorname{sen}\left(k_{n} y\right) e^{i \omega t}
$$

where the eigenvalues are:

$k_{m}=\frac{m \pi}{a}, k_{n}=\frac{n \pi}{b}$

Substituting (3) in (1) we can find the solution as:

$W_{m, n}=\frac{P_{m, n}}{\rho h\left(\omega_{m, n}^{2}-\omega^{2}\right)}$

being $P_{m, n}$ the modal pressure, that is the action given separately by each mode of vibration, $\omega_{\mathrm{m}, \mathrm{n}}$ the natural frequencies of the glass plate as reported in [8]:

$\omega_{m n}=\sqrt{\frac{E I}{\rho h}\left[\left(\frac{m \pi}{a}\right)^{2}+\left(\frac{n \pi}{b}\right)^{2}\right]}$

In the specific case of an incident plane wave [12]:

$P_{m, n}=8 P_{i} I_{m} I_{n}$

where $P_{i}$ is the amplitude of the disturbing wave, contained in: $p_{i}(x, y, t)=P_{i} e^{i \omega t-i k \operatorname{sen} \vartheta_{i} \cos \varphi_{i}-i k \operatorname{sen} \vartheta_{i} \operatorname{sen} \varphi_{i}}$

where $w$ and $k$ are the wave number and phase change of the disturbing wave, $\theta_{i}$ and $\varphi_{i}$ are the incident angles shown in Fig. 3, $I_{m}$ and $I_{n}$ are the coupling constants that have the task to take into account the coupling phenomenon between the fluid and the glass plate. They can be calculated using the following relations:

$I_{m}=\frac{-i}{2} \operatorname{sgn}\left(\sin \vartheta_{i} \cos \varphi_{i}\right)$, if $\_(m \pi)^{2}=\left[\sin \vartheta_{i} \cos \varphi_{i}\left(\frac{\omega a}{c}\right)\right]^{2}$

$I_{m}=\frac{m \pi\left\{1-(-1)^{m} e^{\left[-i \sin \vartheta_{i} \cos \varphi_{i}\left(\frac{\omega a}{c}\right)\right]}\right\}}{(m \pi)^{2}-\left[\sin \vartheta_{i} \cos \varphi_{i}\left(\frac{\omega a}{c}\right)\right]^{2}}$, otherwise

and:

$I_{n}=\frac{-i}{2} \operatorname{sgn}\left(\sin \vartheta_{i} \sin \varphi_{i}\right)$, if $\_(n \pi)^{2}=\left[\sin \vartheta_{i} \sin \varphi_{i}\left(\frac{\omega b}{c}\right)\right]^{2}$

$I_{n}=\frac{n \pi\left\{1-(-1)^{n} e^{\left[-i \sin \vartheta_{i} \sin \varphi_{i}\left(\frac{\omega b}{c}\right)\right]}\right\}}{(n \pi)^{2}-\left[\sin \vartheta_{i} \sin \varphi_{i}\left(\frac{\omega b}{c}\right)\right]^{2}}$, otherwise

being $c$ the wave speed in air; $a, b$ and $\omega$ have the same meaning as stated before. 


\section{AN AUTOMATED CONTROL SYSTEM FOR GLAZED FACADES}

\section{A. Choice of the control system typology}

The ASAC approach is based on the reduction of structural vibrations and radiated sound (transmitted and reflected) as a consequence, by means of actuators bonded on the vibrating surfaces. ASAC can be easily integrated in buildings, as it does not require the use of loudspeakers or error microphones in the receiving environment. However it requires a preliminary study for the selection of suitable actuators to be placed on the vibrating surface under investigation. Various modes of vibration have different radiation efficiencies [8], and some are better coupled to the radiation field than others. This suggests two conclusions:

- only selected modes need to be controlled, rather than the whole response;

- the relative phases and amplitudes of multi-modal response can be adjusted so that their radiated fields interfere destructively.

Moreover, it is necessary to choose the ASAC system controller, which can be either of the feed-forward or feedback type. The feed-forward control [13] requires knowledge of the primary disturbance, which is derived by the use of a reference sensor: in the case of buildings this would require the use of a reference microphone external respect to the building, not feasible for functional and aesthetic issues. Therefore, the feedback type controller seems to be opportune [14]; in the case studied in this paper it will be made up of the following components:

- $\quad$ sensors to detect the vibration (e.g. strain gauges);

- electronic filters to analyze signals from sensors in order to select the component due to the disturbance;

- electronic controller to manipulate signals from the detector;

- secondary actuators to drive the actuators on glazed panels;

- actuator which influence the response of glazed panels.

As shown in Fig. 4, once the signal comes from the sensors, it must be elaborated by charge amplifiers (converting voltage signals into physical variables like displacements, velocity and accelerations), and electronic filters that have a double task:

- they separate the contribution to the total vibration field due to the primary disturbance from that due to secondary sources;

- they compute the radiated field in some positions of the receiving room, to calculate the error (difference between reference value and measured value of the radiated noise), as explained in the following paragraph III.B.

The controller, starting from the error signals, computes the opportune voltage to be supplied to the secondary sources, whose electric power is provided by the amplifiers.

\section{B. The feedback controller}

At this level an algorithm calculates the control voltage to be supplied to the secondary sources. In general it has the task of changing the distributed mass, stiffness and damping of the plate in order to reduce the radiated noise in the internal environment, determined mainly by flexural waves, that needs for this reason to be decreased by the control plant. As stated in subsection III.A, the complexity of the algorithm derives from the fact that reference and control sensors are positioned at the same place, therefore electronic filters are necessary. The controller must opportunely tune its gain coefficients to adjust the properties of the system, so that the radiated noise will be minimized. A number of papers, such as [15], [16], [17] and [18], showed that optimum control is the most suitable for ASAC systems: it has the advantage that the choice in the prescribed change of the dynamic properties is motivated by its aim to reduce structural vibrations or radiated noise. Optimal control systems procedures offer the possibility of designing the controller parameters directly minimizing a cost function of performance which is proportional to the required measure of the system's response. For the sake of computational efficiency it is convenient to define a cost function that is quadratically dependent on the response, simplifying the optimization problem. One of the possible solutions can be the one proposed by [11], where the cost function is given by:

$J=\frac{1}{2 \rho c} \sum_{i=1}^{N}\left|p_{t}^{i}\right|^{2} \Delta S_{i}$

being $J$ the total radiated acoustic power, $\rho$ and $c$ the density and sound velocity of wave in air; $p_{i}$ the sound pressure values measured at some prescribed measurements points and $\Delta S_{i}$ the surfaces relative to each measurement point. Pressure values are not directly measured, but computed from the signals deriving from the reference sensors positioned on the glazed panel (through the use of filters). Thus the structural-acoustic coupling is inherent in the definition of the cost function. It was demonstrated by [11] and [19] that substituting the opportune expression of radiated sound pressure, given by the superposition of the two contributions of both the disturbance and the control actuators, the cost function is scalar. It can be converted in a quadratic expression of complex control voltages, and it was demonstrated that this function has a unique minimum. The general form of the equation to be minimized becomes:

$J=\underline{h}^{T} \underline{q}+\underline{c}^{T} \underline{v}_{i}$

where $\mathbf{q}$ is the vector of complex input disturbances, $\mathbf{h}$ is the vector of transfer functions associated with these disturbances; $\mathbf{c}$ is the control transfer function vector and $\mathbf{v}$ is the unknown vector. In this way a vector of voltages $v_{i}$ is computed, minimizing the total radiated field.

Once the transfer functions between the reference signal deriving from reference sensors and the acoustic radiated

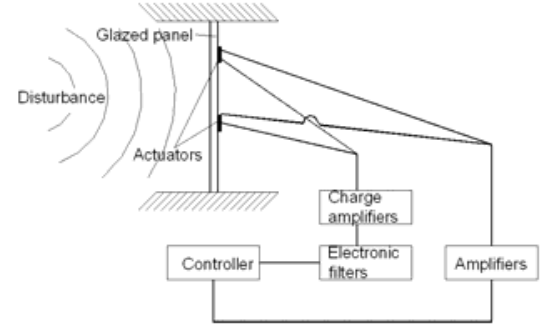

Fig. 4. Scheme of an ASAC system for glazed panels integrated in buildings. 
noise is known for a given system, the control plant will automatically execute all these steps, minimizing the radiated noise even if glazed panels are subject to time-dependent input disturbances, giving back an automated glazed facades, that actively change its properties according to the disturbance. Before implementing this control system, it is necessary to calculate the control transfer functions, which requires as a preliminary stage, the choice of the opportune kind of secondary sources, carried out in the following chapter IV.

\section{THE ACTUATORS}

\section{A. Choice of actuators}

In this chapter the level of controllability of several kinds of actuator on constrained glass panels will be analyzed through numerical simulations. The choice is linked to the possibility of applying control signals on the system in order to guarantee the required control authority for glass panels, on the average very stiff and heavy. However, other issues relative to power consumption, frequency response and physical constraints must be taken into account. References [20] and [21] give a good review of the many kinds of actuators on the market. As already stated in Chapter I, some successfully tests were carried out, using PZT patches as actuators for active control of vibrations of airplane fuselages [5], [6]. In [22] the use of stack actuators was suggested for the control of active vibrations of stiff plates. In this chapter the performances of PZT patches and stack actuators are compared, as they gave good performances in previous experimental test [5], [6], [22]. Transparency of glazed panels can be preserved by positioning actuators along the edge and hidden by the frame if necessary for image purposes. In Fig. 5 one of the possible configurations is shown, where actuators are placed along two of the four sides, to perform the control of such 2D structures. Some technological problems are connected with the use of stack actuators, as they need a reaction mass or system to generate the necessary secondary force: the rigidity of the reaction system must be comparable with that of the actuators when high forces must be provided. As detailed in the following paragraph, the authors suggest applying such actuators on panels using as reaction system a stiffener that could be bonded directly to the glass or to the frame of facades.

\section{B. Numerical model for the simulation of glass plates} behavior excited by actuators

Fig. 6 shows three different configurations for the suggested

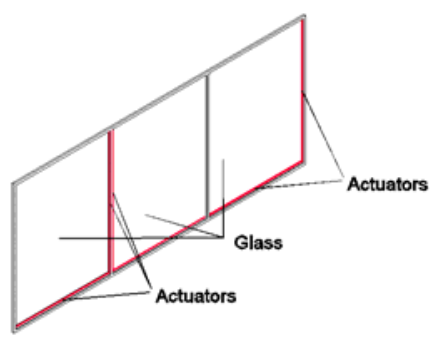

Fig. 5. Possible positioning of actuators on a portion of a glazed facade. disposals for actuators, analyzed hereon:

- $\quad$ asymmetric PZT patches (Fig. 6-a);

- stack PZT actuators with reaction system fixed on the frame of the facade (Fig. 6-b);

- stack PZT actuators with reaction system fixed on the glass panel (Fig. 6-c).

In the asymmetric disposal of Fig. 6-a, the PZT patch excites the 2D structure with pure bending, that can be simulated with the numerical model developed in [23]. In this paper it is assumed that the strain slope is continuous through the thickness of the glass plate and of the PZT patch, but different along the directions parallel to the plate sides, that in turn are assumed parallel to the coordinate axes (the strain slopes are billed $C_{x}$ and $C_{y}$ ). The mathematical relation between strain and $z$-coordinate is:

$\varepsilon_{x}=C_{x} z$

$\varepsilon_{y}=C_{y} z$

being the origin of the $z$-axis in the middle of the plate thickness and $\varepsilon$ the strain. The unconstrained strain of the actuator $\left(\varepsilon_{\mathrm{pe}}\right)$ along plate axes is dependent to the voltage applied $(V)$, the actuator thickness $\left(h_{a}\right)$ and the PZT strain constant along $x$ or $y$ directions $\left(d_{x}=d_{y}\right)$ :

$\varepsilon_{p e}=\frac{d_{x} V}{h_{a}}$

Considering that the plate is subject to pure bending, no longitudinal waves will be excited, and by applying the moment equilibrium condition about the centre of the plate along $x$ and $y$ directions as in [11], a strain dependent relation is written:

$C_{x}=C_{y}=C=K^{f} \varepsilon_{p e}$

where the material-geometric constant is computed by:

$$
K^{f}=\frac{3 E_{p e}\left[\left(h_{b}+h_{a}\right)^{2}-h_{b}^{2}\right]\left(1-v_{p}\right)}{2 E_{p e}\left[\left(h_{b}+h_{a}\right)^{2}-h_{b}^{3}\right]\left(1-v_{p}\right)+2 E_{p} h_{b}^{3}\left(1-v_{p e}\right)}
$$

The plate thickness is $2 h_{b}$, the plate elastic modulus is $E_{p}$, the actuator elastic modulus is $E_{p e}$, and $v_{p}$ and $v_{p e}$ are the Poisson coefficients of the plate and actuators respectively. Assuming that the moments induced in the $x$ and $y$ directions (billed with $m_{x}$ and $m_{y}$ ) are present only under the PZT patch, and assuming that it is located between the points of coordinates $\left(x_{1}, y_{1}\right)$ and $\left(x_{2}, y_{2}\right)$, in [11] it is shown that:

$m_{x}=m_{y}=C \varepsilon_{p e}\left[H\left(x-x_{1}\right)-H\left(x-x_{2}\right)\right]\left[H\left(y-y_{1}\right)-H\left(y-y_{2}\right)\right]$

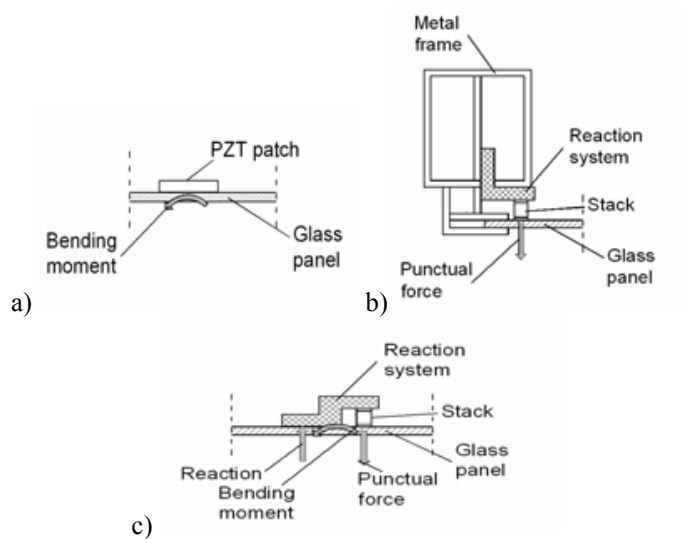

Fig. 6. Actuators positioned on a glass panel: a) PZT patches; b) stack; c) stack with angle stiffener. 
being $H(x)$ the Heaviside function [24] and $C=E I K^{f}$, where $I$ is the moment of inertia of the plate. The equation of motion for plates subject to flexural waves can be written [11]:

$$
E I\left(\frac{\partial^{4} w}{\partial x^{4}}+\frac{\partial^{4} w}{\partial y^{4}}\right)+\rho h \frac{\partial^{2} w}{\partial t^{2}}=-p(x, y)
$$

where $p$ is an external uniform pressure applied on the plate. Equation (16), if written with the actuator induced moment, becomes [11]:

$$
\frac{\partial^{2}\left[M_{x}(x)-m_{x}(x)\right]}{\partial x^{2}}+\frac{\partial^{2}\left[M_{y}(y)-m_{y}(y)\right]}{\partial y^{2}}-\omega^{2} \rho S w=0
$$

where $M$ is the internal plate moment and $m$ is the actuator induced bending moment; $\rho$ and $S$ are density and surface of the plate; $w$ is the displacement and $\omega$ is the wave phase change. Assuming that the actuator is perfectly bonded on the glass plate and substituting (16) inside (18), the following inhomogeneous plate equation is obtained [11]:

$$
\begin{aligned}
& E I \nabla^{4} w+m^{\prime \prime} \frac{\partial^{2} w}{\partial t^{2}}=C_{0} \varepsilon_{p e}\left[\delta^{\prime}\left(x-x_{1}\right)-\delta^{\prime}\left(x-x_{2}\right)\right] . \\
& \cdot\left[H\left(y-y_{1}\right)-H\left(y-y_{2}\right)\right]+C_{0} \varepsilon_{p e}\left[H\left(x-x_{1}\right)-H\left(x-x_{2}\right)\right] . \\
& \cdot\left[\delta^{\prime}\left(y-y_{1}\right)-\delta^{\prime}\left(y-y_{2}\right)\right]
\end{aligned}
$$

where $E I$ is the stiffness of the plate, $\delta$ is the derivative of the Dirac delta function [24], $H$ is the Heaviside function. The solution of (17) can be calculated by using the modal expansion of (3), which gives back:

$$
W_{m n}=\frac{4 C_{0} \varepsilon_{p e}}{\rho h m n \pi^{2}\left(\omega_{m n}^{2}-\omega^{2}\right)}\left(k_{m}^{2}+k_{n}^{2}\right) p_{1} p_{2}
$$

where: $p_{1}=\cos \left(k_{m} x_{1}\right)-\cos \left(k_{m} x_{2}\right), p_{2}=\cos \left(k_{n} y_{1}\right)-\cos \left(k_{n} y_{2}\right)$.

Equation (20) can be written in terms of (5), defining the variable:

$$
P_{m n}=\frac{4 C_{0} \varepsilon_{p e}}{m n \pi^{2}}\left(k_{m}^{2}+k_{n}^{2}\right) p_{1} p_{2}
$$

Thus, given the properties of the PZT patches under use and the ones of the plate, (20) together with (5) and (3) gives back the transversal displacement function on the 2D plate caused by PZT patch actuators with respect to $x$ and $y$ coordinates.

In the case shown in Fig. 6-b, the stack actuator has the task of providing a punctual force, instead of a bending moment. Following a procedure similar to the one explained in this subsection, it is possible to calculate a numerical model [11] that describes the vibration field in terms of (3) and (5) exploiting the following relation:

$$
P_{m n}=\frac{4 F_{a}}{a b} \sin k_{m} x_{f} \sin k_{n} y_{f}
$$

where $a$ and $b$ are the side lengths of the plate; $x_{f}$ and $y_{f}$ are the coordinate of the point where the force $F_{a}$ is applied, that is the action provided by the stack actuator, which is dependent to the reaction system stiffness. Assuming $d_{z}$ the strain constant of the actuator along the z-direction, its unconstrained displacement will be computed by [11]:

$w_{a}=\frac{d_{z} V}{L_{a}}$

where $L$ is its height. In fact the real displacement of the stack is lower than (23) because the reaction system has finite stiffness $K$, and the force effectively exerted by the stack along the $z$-direction is:

$$
F_{a}=\frac{d_{z} V K}{1+\frac{K}{K_{a}}}
$$

being $k_{a}$ the actuator stiffness. Equation (24) gives the force really transferred by the stack to the glass panel, which is a fraction of the total force in the case of $k=\infty$ :

$F_{\text {max }}=d_{z} K_{a} V$

As in the previous case, the transverse vibration displacement of a 2D plate can be calculated by (22) with (5) and (3).

In the case of Fig. 6-c, stack actuators are fixed by means of an angle stiffener firmly bonded to the glazed plate, and the voltage provided to the stack will cause a force at the base of the actuator, and a reaction force and a moment at the base of the stiffener, as shown in Fig. 6-c. Considering the high complexity of this system, due to the many actions superimposing at the stack level, a Finite Element Analysis was carried out, in order to study the local stress field generated by the fixing of the angle stiffener on glazed plates also. It is assumed that angle stiffeners are fixed in a way capable of conferring the requested rigidity to the system.

\section{Numerical analyses of the level of controllability of glass plates}

Buildings' glazed facades are often subject to broadband disturbance with important contribution of low frequency waves, between 60 and $500 \mathrm{~Hz}$, where is contained their resonance frequency. In the following numerical simulations, the disturbance is assumed to be a wave with frequency near the second mode of vibration $(2,2)$ of such panels, whose effect is compared with the one given by the use of the three different kinds of actuator listed in IV.B. The glazed panel is supposed to be simply supported along the edges. The three different configurations of Fig. 6 are studied numerically: for cases 6-a and 6-b numerical algorithms (detailed in IV.B) are implemented with MatLab 6.5; for case 6-c a Finite Element Analysis is carried out with the commercially available software COSMOS/M 2.7. The properties of the glazed plate used for these simulations are listed in Table I, while for PZT patches in Table II. For the simply supported plates of Table I, natural frequencies of vibration are given by (6), whose results are listed in Table III for the smallest modes; so the frequency of the disturbance was chosen equal to $78 \mathrm{~Hz}$.

In the first case of Fig. 6-a, the behavior of the panel of Table I is simulated when equipped with two dispositions of PZT patches:

- 8 patches equally distributed $0.05 \mathrm{~m}$ far from the panel edges;

- 26 patches equally distributed $0.05 \mathrm{~m}$ far from the panel edges.

Each rectangular shaped patch measures 0.05 by $0.04 \mathrm{~m}$. Fig. 7 shows the distribution of the maximum amplitude vibration field along the middle axis of the plate, computed by fixing $y=1 / 2$ in (3). One of the diagrams is referred to the effect due to the disturbance wave at frequency $v=78 \mathrm{~Hz}$ and intensity $100 \mathrm{~dB}$, computed from (3) and (5). For a voltage of $150 \mathrm{~V}$ (that is the highest limit for low-voltage actuators) PZT patches can generate vibration fields far lower than the one generated by the disturbance. 


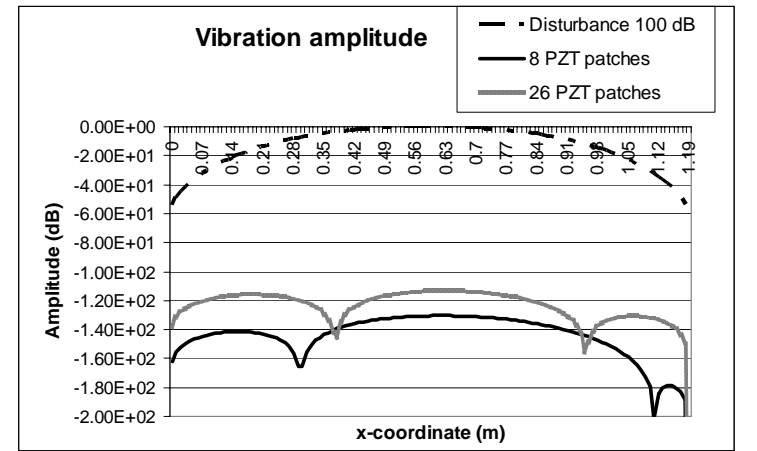

Fig. 7. Amplitude displacement along the $y=1 / 2$ axis due to the positioning of PZT patches actuators, normalized with respect to the maximum disturbance value.

As second case, the solution of Fig. 6-c is studied hereon. An angle stiffener is assumed to be bonded to the glazed surface; table IV lists the cases object of the Finite Element analysis, varying some parameters in the configuration of the system. Each simulation was carried out using a 2D finite element model of the system made up of glass panel and angle stiffener along one of the two symmetry axes, subdivided in square shaped finite elements of $0.001 \mathrm{~m}$ side length. The aim of the simulation is analyzing the level of controllability of such stack actuators on glass panels. It is assumed that the panel is equipped with three stack actuators per each side, so that the central ones act on a strip $0.4 \mathrm{~m}$ wide. The first study was aimed at verifying that the stress field is inside the acceptable range. Fig. 8 shows that the maximum stress values were lower than the acceptable limits $\left(2.4 \cdot 10^{8} \mathrm{~N} / \mathrm{m}^{2}\right.$ for Steel Fe 510 and $1.6 \cdot 10^{7} \mathrm{~N} / \mathrm{m}^{2}$ for glass), being in the worst two cases $\mathrm{n} .4$ and $\mathrm{n} .7$ equal to $3.9 \cdot 10^{5} \mathrm{~N} / \mathrm{m}^{2}$ and $5.7 \cdot 10^{5} \mathrm{~N} / \mathrm{m}^{2}$. These values are obtained in the node common to the angle stiffener and glass plate where the stress field is maximum. As can be inferred by Fig. 9 stack actuators have high controllability on the plate vibrations, even if they generate a

TABLE I

GLAZED PLATE PROPERTIES

\begin{tabular}{cccc}
\hline \hline Symbol & Quantity & $\begin{array}{c}\text { Units of } \\
\text { measurement }\end{array}$ & Value \\
\hline $\mathrm{E}_{\mathrm{p}}$ & Modulus of elasticity & $\mathrm{Pa}$ & $6.9 \cdot 10^{10}$ \\
$v_{p}$ & Poisson coefficient & - & 0.23 \\
$\rho_{p}$ & density & $\mathrm{Kg} / \mathrm{m}^{3}$ & 2457 \\
$h_{p}$ & thickness & $\mathrm{m}$ & 0.006 \\
$l_{p}$ & Side length & $\mathrm{m}$ & 1.2
\end{tabular}

TABLE II

PZT PATCHE'S PROPERTIES

\begin{tabular}{cccc}
\hline \hline Symbol & Quantity & $\begin{array}{c}\text { Units of } \\
\text { measurem. }\end{array}$ & Value \\
\hline $\mathrm{E}_{\mathrm{pe}}$ & Modulus of elasticity & $\mathrm{Pa}$ & $6.3 \cdot 10^{10}$ \\
$v_{p e}$ & Poisson coefficient & & 0.3 \\
$\rho_{p e}$ & density & $\mathrm{Kg} / \mathrm{m}^{3}$ & 7650 \\
$h_{p e}$ & thickness & $\mathrm{m}$ & 0.0002 \\
$d_{31}$ & Expansion constant & $\mathrm{m} / \mathrm{V}$ & -0.000000000166
\end{tabular}

TABLE III

NATURAL FREQUENCIES OF VIBRATION

\begin{tabular}{cccc}
\hline \hline Mode & Frequency $(\mathrm{Hz})$ & Mode & $\begin{array}{c}\text { Frequency } \\
(\mathrm{Hz})\end{array}$ \\
\hline$(1,1)$ & 20.6 & $(2,2)$ & 82.4 \\
$(1,2)$ & 51.5 & $(2,3)$ & 133.8 \\
$(1,3)$ & 102.9 & $(3,3)$ & 185.2
\end{tabular}

TABLE IV

ANGLE STIFFENER BONDED TO THE GLAZED PANEL

\begin{tabular}{cccccc}
\hline \hline $\begin{array}{c}\text { Case } \\
\text { ID }\end{array}$ & $\begin{array}{c}\text { Angle } \\
\text { stiffener } \\
\text { material }\end{array}$ & $\begin{array}{c}\text { Angle } \\
\text { stiffener } \\
\text { thickness } \\
(\mathrm{m})\end{array}$ & $\begin{array}{c}\text { Stack } \\
\text { height } \\
(\mathrm{m})\end{array}$ & $\begin{array}{c}\text { Stack } \\
\text { section } \\
\left(\mathrm{m}^{2}\right)\end{array}$ & $\begin{array}{c}\text { Actuator } \\
\text { Force (N) }\end{array}$ \\
\hline 1 & Steel & 0.01 & 0.02 & $7.8 \cdot 10^{-5}$ & 15 \\
2 & Steel & 0.01 & 0.02 & $7.8 \cdot 10^{-5}$ & 25 \\
3 & Steel & 0.02 & 0.04 & $7.8 \cdot 10^{-5}$ & 15 \\
4 & Steel & 0.02 & 0.04 & $7.8 \cdot 10^{-5}$ & 35 \\
5 & Glass & 0.01 & 0.02 & $7.8 \cdot 10^{-5}$ & 15 \\
6 & Glass & 0.02 & 0.04 & $7.8 \cdot 10^{-5}$ & 35 \\
7 & Glass & 0.02 & 0.04 & $7.8 \cdot 10^{-5}$ & 50
\end{tabular}

disturbance effect near the position of the angle stiffener. Therefore, further analyses will be carried out to evaluate the effect of the superposition between the acoustic field radiated by the portion of the plate near the actuators and the rest of the plate. The stiffness of the reaction system provided by the angle stiffener bonded on the glass is computed for some cases, by the knowledge of displacements at the points of application and the values of the forces there applied:

- for case n. 1 it is $\mathrm{k}=136 \mathrm{~N} / \mu \mathrm{m}$;

- for case n. 5 it is $\mathrm{k}=186 \mathrm{~N} / \mu \mathrm{m}$;

- for case $n .6$ it is $\mathrm{k}=42 \mathrm{~N} / \mu \mathrm{m}$.

A rigid stiffener can produce very efficient systems, but in this case angle stiffeners must have high thickness.

In the third case vibration amplitudes are computed for the stack configuration shown in Fig. 6-b. In Fig. 10 such vibration amplitudes are drawn with dependence to the voltage provided to stack actuators. It is assumed that the panel is equipped with 3 actuators $\left(0.02 \mathrm{~m}\right.$ long with $7.8 \cdot 10^{-5}$ $\mathrm{m}^{2}$ cross sectional area) per each side, equally spaced and at a $0.03 \mathrm{~m}$ distance from the two edges; the stiffness of the reaction system (frame) is assumed equal to $200 \mathrm{~N} / \mu \mathrm{m}$. Fig. 10 shows that, regardless of the small rigidity of the reaction system, the stack actuators can produce a vibration amplitude comparable with the one due to the disturbance with only a voltage of $100 \mathrm{~V}$.

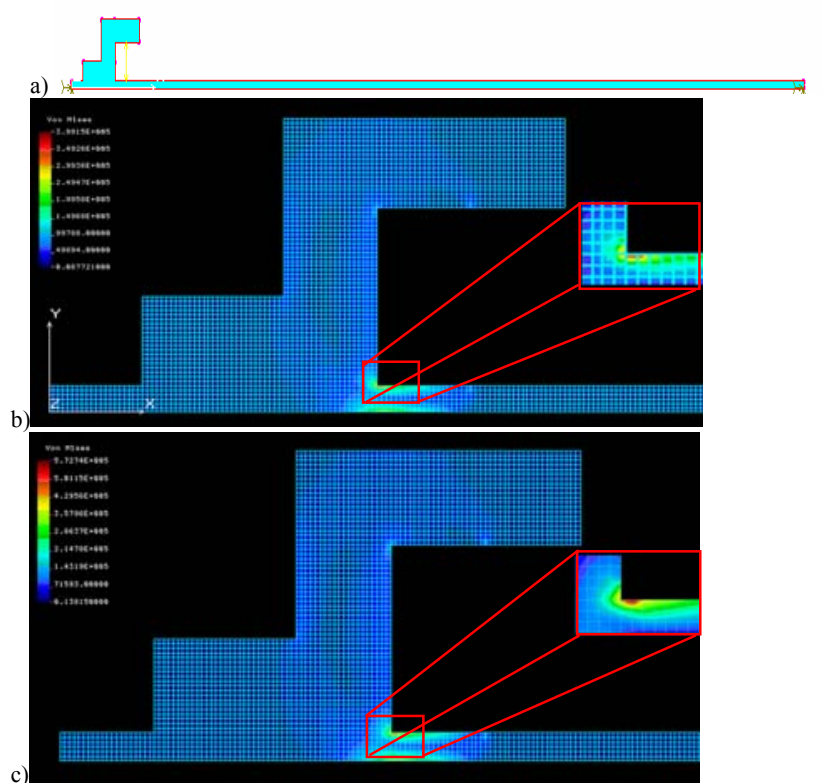

Fig. 8. Geometry of the Finite Element model (a), its stress field for case n. 4 (b) and case n. 7 (c) near the angle stiffener. 


\section{CONCLUDING REMARKS AND FUTURE RESEARCH DEVELOPMENTS}

As shown in this contribution stack actuators can constitute suitable transducers for controlling 2D glazed plate vibrations. High performances are obtained with the use of angle stiffeners bonded to the glazed panel, even if further analyses must be carried out in order to study suitable methods for fixing these on plates. Moreover, other experimental or numerical tests will be planned, in order to evaluate the acoustic coupling effect originated by vibrations of the portion of glazed plate near the angle stiffener. The next two research steps will be aimed at:

- validation of a numerical procedure for the evaluation of the acoustic field radiated by a vibrating glass plate, necessary to calculate the disturbance transfer functions $\boldsymbol{h}$ firstly cited in (11);

- implementation of the control algorithm already described in Chapter III, on a system made up of a glazed plate and stack actuators, in order to test the performance which can be obtained in the cancellation of disturbing waves.

Given the high flexibility of the system and its effectiveness at low frequencies it could confer to glazed facades, properties similar to the ones of massive $2 \mathrm{D}$ structure without improving loads on the bearing frame of buildings.

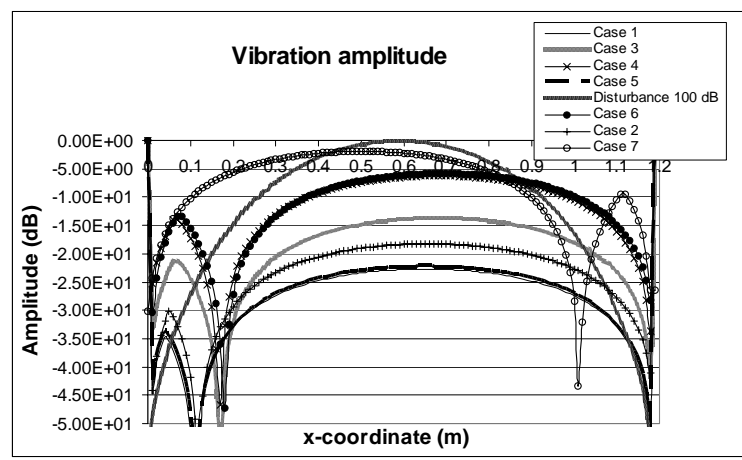

Fig. 9. Amplitude displacement along the $\mathrm{y}=1 / 2$ axis due to the positioning of stack with stiffener bonded on the plate, normalized with respect to the maximum disturbance value.

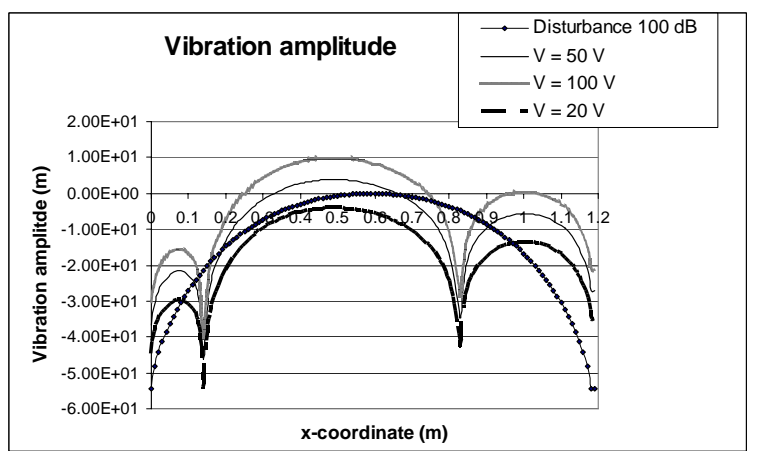

Fig. 10. Amplitude displacement along the $y=1 / 2$ axis due to the positioning of stack with stiffener fixed to the frame, normalized with respect to the maximum disturbance value.

\section{REFERENCES}

[1] De Man, P., Francois, A., Preumont, A., "Active control of noise transmission through double wall structures, an overview of possible approaches", $6^{\text {th }}$ National Congress on Theoretical and Applied Mechanics, Ghent, May 26-27, 2003.

[2] Kaiser, O. E., Pietrzko, S. J., Morari, M., "Feedback control of sound transmission through a double glazed window", Journal of Sound and Vibration, 263, pp. 775-795, 2003.

[3] De Fonseca, P., Dehandschutter, W., Sas, P., Van Brussel, H., "Implementation of an active noise control system in a double-glazing window", Proceedings of ISMA 21, Noise and Vibration Engineering Conference, Leuven, Vol. 1, pp. 377-388, 1996.

[4] Pan, J., Bao, C., "Analytical study of different approaches for active control of sound transmission through double walls", Journal of the Acoustical Society of America, 103(2), pp. 1664-1670, 1997.

[5] Simpson, M. A., Luong, T. M., Fuller, C. R., Jones, J. D., "Full-scale demonstration of cabin noise reduction using active vibration control", Journal of Aircraft, Vol. 28, n. 3, pp. 208-215, 1991.

[6] Fuller, C. R., Snyder, S. D., Hansen, C. H., Silcox, R. J., ”Active control of interior noise in model aircraft fuselages using piezoceramic actuators", AIAA Journal, Vol. 30, n. 11, pp. 2613-2617, 1992.

[7] Fuller, C. R., Gibbs, G. P., "Active control of interior noise in business jet using piezoceramic actuators", Proceedings of Noise-Con 94, pp. 389-394, 1994.

[8] Fahy, F., "Sound and structural vibration - radiation, transmission and response", Academic Press, London - San Diego - New York - Berkeley - Boston - Sydney - Tokyo - Toronto, par. 4.3, 1985.

[9] "Technical Report WP 1.1: Source modeling of road vehicles" , EC project funded: IMAGINE - Improved Methods for Assessment of the Generic Impact of Noise in the Environment", 2003.

[10] Hall, D. E., "Basic Acoustics", John Wiley and sons, New York Chichester - Brisbane - Toronto - Singapore, pp. 122-124, 1987.

[11] Fuller, C. R., Elliott, S. J., Nelson, P. A., "Active Control of Vibration", 2nd Edition, Academic Press, San Diego-London- Boston-New York, par. $2.10-8.4-8.5,1997$.

[12] Roussos, L. A., "Noise transmission loss of a rectangular plate in an infinite baffle", NASA, TR 2398, Washington, DC, 1985.

[13] Isermann, R., "Digital control system", Springler-Verlag, BerlinHeidelber-New York, pp. 302-308, 1981.

[14] Franklin, G., Powell, J. D., Emami-Naeini, A., "Feedback control of dynamic systems", Addison-Wesley Publishing Company, pp. 87- 117, 1988.

[15] Baumann, W. T., Ho, F. S., Robertshaw, H. H., "Active structural acoustic control of broadband disturbances", Journal of the Acoustic Society of America, 92(4), 1992.

[16] Cunnefare, K. A., "The minimum multimodal radiation efficiency of baffled finite beams", Journal of the Acoustic Society of America, 90(5), 1991.

[17] Baumann, W. T., Saunders, W. R., Robertshaw, H. H., "Active suppression of acoustic radiation from impulsively excited structures", Journal of the Acoustic Society of America, 90(6), 1991.

[18] Pan, J., Snyder, S. D., Hansen, C. H., Fuller, C., R., "Active control of far-field sound radiated by a rectangular panel - A general analysis", Journal of the Acoustic Society of America, 91(4), 1992.

[19] Nelson, P. A., Elliott, S. J., “Active control of sound", Academic Press, London, pp. 183-188, 1992

[20] Broch, J. T., "Mechanical Vibration and shock measurements", Bruel \& Kjaer, Denmark, 1984.

[21] Cremer, L., Heckl, M., "Structure-born sound", $2^{\text {nd }}$ edition, SpringerVerlag, Berlin, 1988.

[22] Andrew, J. Y., "Active control of vibration in stiffened structures", $\mathrm{PhD}$ Thesis, Department of Mechanical Engineering, The University of Adelaide, 1995.

[23] Dimitriadis, E.K., Fuller, C. R., Rogers, C.A., "Piezoelectric actuators for distributed vibration excitation of thin plates", American Society of Mechanical Engineers Journal of Vibration and Acoustics, 113, pp. 100$107,1991$.

[24] Spanier, J., Oldham, K. B., "An atlas of functions", Washington D.C., Hemisphere, pp. 63-69, pp. 79-82, 1987. 